\title{
Investigating possible fraudulent activity at a research site
}

\author{
Carol Knott ${ }^{*}$, Joanne Henderson, Louise Bowman, Martin Landray \\ From 3rd International Clinical Trials Methodology Conference \\ Glasgow, UK. 16-17 November 2015
}

\section{Background}

A risk-based approach to monitoring clinical trials aims to detect non-compliance with the protocol or regulatory requirements that may compromise the participants' well-being or the trial's ability to produce reliable results.

\section{Method}

We describe a pragmatic framework for investigating and evaluating suspicions of poor performance/practice and the steps to be taken if findings suggest fraudulent activity.

Prior to site audit:

- Check reliability of information that prompted concerns

- Prepare dossier of site information, detailing suspect data points

- Arrange visit promptly (but without indicating concerns)

\section{During audit:}

- Two auditors: one to ask open questions, the other as witness and scribe.

- Interview staff individually, establishing their knowledge of trial procedures and incident. Note conflicting information.

- Establish key facts:

1. Were participants real and eligible?

2. Did they consent?

3. Are test/clinical measurements/data valid?

4. Was appropriate treatment/intervention given?

5 . Was follow-up provided, were events reported?

6. Are participants safe and data reliable?
- Obtain documentary evidence, maintaining confidentiality of trial participants

- Document findings

After audit:

- Implement corrective actions to:

1. Ensure safety of participants

2. Address important deficiencies in data quality

3. Support site: training, additional monitoring/ support, replace staff.

- Look for systemic trial quality issues e.g. other staff, other data

1. Apply lessons/corrective actions to the whole trial

- Notify appropriate parties: Sponsor, steering committee, data monitoring committee, regulatory body, ethics board, host institution, funder, and professional bodies.

\section{Conclusion}

Cases of serious misconduct or fraud do occur. Taking a systematic approach to an investigation ensures appropriate action is taken to preserve study intergrity.

Published: 16 November 2015

doi:10.1186/1745-6215-16-S2-P193

Cite this article as: Knott et al:: Investigating possible fraudulent activity

at a research site. Trials 2015 16(Suppl 2):P193. 